\section{UNDERSTANDING NECESSITY CAUSALLY ${ }^{1}$}

\section{Puttawit Bunnag $^{2}$}

\begin{abstract}
The paper concerns a central issue of alethic modality. It attempts to provide a criterion of meaning for statements with modal words: 'necessary', 'possibly', 'must', 'can', 'could', etc. By considering the main problems concerning modal logic, logic dealing specifically with modal language, the paper chooses to understand the meanings of modal language by 'possible worlds' semantics, and tries to make it more credible by employing a concept of causality which underline most of our normal modal language. Furthermore, the paper attempts to answer the following questions: Why are formal expositions essential to philosophically understanding problematic modal discourses?; What is the conceptual burden they impose on us which needs to be overcome?; Why are existing philosophical endeavours taking part in this semantic contest 'unsatisfactory' or 'inadequate'?; How can we understand modal discourses causally?
\end{abstract}

\footnotetext{
1 I am grateful to Prof. Mark Tamthai for supervising my dissertation on which this paper is based, Dr. Richard Dyche who inspired me to research this topic, and Dr. Soraj Hongladarom for important comments and suggestions.

2 Lecturer, Department of Philosophy and Religion, Srinakharinwirot University; graduate student, Department of Philosophy, Chulalongkorn University, Bangkok, Thailand.
}

\section{Introduction}

This paper focuses on alethic modalities. Broadly speaking, it is about the truth involving necessity and possibility. More narrowly, it tries to give a criterion of meaning for statements with modal words, that is, words such as 'necessary', 'possibly', 'must', 'can', 'could' and so on. Before considering the logical side of the main problems with which this paper is concerned, I would like in the introduction to present an idea about modal concepts in a somewhat informal style.

We cannot deny that it seems natural to allow various modal words to be used in our everyday speech, though, seemingly, we can imagine that our lives would not be changed so much if we, from now on, abstained from using those modal words. However, we all agree that it is possible for the sheet of paper that you are looking at right now to be darker than it is; or that the statement " The tallest female in the world is the same person as the tallest male" is false necessarily. But if someone asks you to tell her what is the fact that your belief about the sheet of paper just mentioned intends to represent, or what is definitely meant by the statement above about tallest persons, you might find that it is not easy to give an answer without, at least, a little awkwardness. For there is more than one way to response to the questions; and they do not seem to stop us from posing further questions.

For example, in case you believe that this sheet of paper could have been darker than it actually is, your response might be that it expresses your negative belief about the necessity of this paper to be in a certain color. If you are asked further "What makes you believe the answer you just gave?", and you bring your initial belief 
that the paper could turn darker to be the answer, then you are responding to the question about possibility with an answer about necessity, and vice versa. As we will see later, if you think that this kind of answer alone is sufficient to avoid any further inquiry about the belief in question, it means that you are accepting that modality can satisfactorily be grounded on certain rules of language or logic. And you will see, from what we will consider later, that this leads to a serious problem, central to the interest of this paper.

The other line of response to questions of the ground for believing in modal statements does not enclose itself with a fence of synonymies, as we saw in the previous paragraph; it tries, rather, to give more fundamental meanings to modal speech. Philosophically speaking, it offers what is generally called 'semantics' to any unit of our language concerning modality. In regard to positions towards the meanings of modal discourses, we find a variety of ways to specify those meanings. Notably among them is a view which treats 'modal talk' as talk about 'possible worlds'. We will discuss motives behind this idea in the next section. For now, it should be noted that a major problem of this 'possible world' semantics is its credibility of insuring us against suspecting whether the expositions it offers could be called 'suitable' or 'adequate' in honestly representing our pre-philosophical understanding about the nature of modal language.

The main purpose of this paper is to propose a way to understand the meanings of modal language by 'possible worlds' semantics; but try to make it more credible by employing a concept of causality which I believe underlines most, if not all, of our normal modal talk. In the following sections I will address the following questions: Why are formal expositions essential to philosophically understanding problematic modal discourses in our activities of communication?; What is the conceptual burden they impose on us which needs to be overcome?; Why are existing philosophical endeavours taking part in this semantic contest 'unsatisfactory' or 'inadequate'?; and finally, How can we understand modal discourses causally, and in what manner does this kind of understanding save us from many conceptual predicaments?

\section{Modal logics and its problems}

For getting rid of conceptual difficulties in formal logic concerning the so-called 'material implication', C. S. Lewis, in 1918, invented modal logic in order to make a distinction between two kinds of implication (Cf. Lewis and Langford, 1959). Before, ordinary statements in "if...then..." form were represented by standard sentential logic with the material implication symbol ' $\rightarrow$ ' as " $p \rightarrow q$ ", and the only situation making them false is one where $p$ is true and $\mathrm{q}$ is false. Its main problem is that it seems not to neatly represent "if...then..." statements as we ordinarily use them in our natural languages. This specification of meaning has brought with it a number of paradoxes, such as that we can construct a 'true' if-then sentence, even though neither of its two parts is true: "If Mr. Smith were a woman, he could become pregnant; or the subject matters of its connected sentences are never related at all: "If Michelangelo is a great artist, Thailand is now a developing country". So Lewis proposed a logical symbol, represented here as ' $\Rightarrow$ ', called 'strict implication', intending it to more satisfactorily translate our ordinary use of that kind of problematic statement. $\mathrm{He}$ 
defined ' $\Rightarrow$ ' in terms of another symbol ' $\square$ ', which will be examined more closely below; and this has made the material implication relation become a necessary relation. The paradoxes above, then, can be made to disappear by using the strict implication, rather than the material one, to represent our ordinary if-then statements.

In order to deal with modal notions contained in our normal use of language, certain new units of formal language were developed; and, simultaneously, were constructed, with the technical assistance of standard formal logic, to become what we now call 'modal logic'. We could thus comprehend modal logic as an attempt to make meanings of our natural language statements involving modality be so formal as to rigidly identify their truth and falsehood, along with the inferential relations among them.

However, the manner in which modal logic makes sense of modal statements is rather like fencing these statements in some kind of literal definitions, or in synonymies as mentioned in the introduction, hence fencing them off from further explications. When we say "Eva is possibly on the plane to Phuket.", given that $\mathbf{p}$ represents "Eva is on the plane to Phuket", modal logic would translate it as " $\bullet \mathbf{p}$ ", and equate it to " $\square \sim \mathbf{p}$ ", which means "It is not the case that Eva is necessarily not on the plane to Phuket". On the other hand, if you say "Eva is necessarily on the plane to Phuket", this kind of logic could translate it as " $\square$ " or " $\sim \sim \mathbf{p}$ ", which means "It is not the case that Eva is possibly not on the plane to Phuket". Apart from giving us more precise configurations of our normal modal statement with which we probably are confused, plus the apparatus of standard formal logic in any level, it also equips us with a procedure to grasp and judge whether a statement can be correctly inferred from a given modal statement, or from a group of statements. We must, however, acknowledge the different varieties of modal logic systems, based on different sets of axioms or rules of inference. These several nonequivalent modal systems have brought about various ways to make a judgment whether a certain modal statement can be correctly inferred from, or to, another one.

For example, a system called $\boldsymbol{T}$ (or $\boldsymbol{M}$ ) uses two axioms: (1) $\square \mathbf{p} \rightarrow \mathbf{p}$ and (2) $\square(\mathbf{p} \rightarrow \mathbf{q})$ $\rightarrow(\square \mathbf{p} \rightarrow \square \mathbf{q})$; and it defines ' $\vee$ ' by ' $\sim \square \sim$ ' as we have seen. According to this system, if a statement is necessarily true then we can correctly say by the axiom (1) that it is the case, or, it is just true. This axiom reflects some sense of our common understanding and use of 'necessarily', that whatever is necessarily so is so. Nevertheless, in a system called $\boldsymbol{K}$, with only axiom (2) as its single axiom, we can not validly express this sense of necessity. For the formula $\square \mathbf{p} \rightarrow \mathbf{p}$ is not a theorem of $\boldsymbol{K}$, say, it can not be proved in $\boldsymbol{K}$. Consequently, we can not validly state from $\boldsymbol{K}$ something the first axiom of $\boldsymbol{T}$ tries to state. This is only just one case of inequivalence between different modal logic systems. ${ }^{3} \quad$ It seems that at only the surface of their axiomatizations, without more semantically clarification, it is hard for us to definitely tell which system is the one we should accept.

The situation above might induce one to search for a way to choose the most plausible system among many. Nevertheless, it would appear impossible to find one system unless we find a way of

\footnotetext{
${ }^{3}$ You can see more instances of this kind of problem in Hughes and Cresswell, 1996.
} 
making clear what all of their units of language are commonly referring to, by which we could understand ,for instance, the exact meaning of ' $\nabla$ ' and ' $\square$ ' for all systems. However, had the ideas we have about modality remained stacked within only those relations among interdefinable symbols, our hope to make sense of those modal systems on more than an intuitive basis would continue to be in vain. Fortunately, in the early 1960s there was a breakthrough in the study of the semantics of those logical systems. Devices of a discipline called 'model theory', using abstract mathematical structures to interpret formal systems, were developed as to specify certain conditions to be satisfied for things to be as a statement says they are. These formal conditions, or models, would be comprehended as the meanings of the modal statements (Cf. for example, Kripke, 1971, Hintikka, 1979).

A number of philosophers specified the models in question with sets, or domains, of worlds. We could call them 'possible worlds'; for their domains are not restricted to things in the actual world. As a result, we could understand that a necessary statement is one that is true in all possible worlds; a possibly true statement is one that is true in at least one possible world; a contingently true statement is one that is true in this world, but not in all worlds; and the impossible statement as one never true in any world, provided that we are able to deal successfully with understanding the meaning of 'true in a world' (Cf. Loux, 1979). Consequently, ' $\diamond$ ' and ' $\square$ ' used in various modal logic systems have been illuminated by that same light; and different sets of axioms formerly never reconcilable on the same semantic basis have begun to be understandable as to why they have been accepted differently by different systems. Further technical discussion about these formal achievements need not concern us here. But I would like to conclude here that the answer which model-theoretic possible worlds theories can give to nonequivalences amongst modal systems is that these modal systems, apart from their procession of different sets of axioms, have a difference in the kind of essential element for interpreting them as model-theoretic systems. The mentioned element is a relation which each of the entities, such as 'worlds' or 'objects' (in those worlds), has to be in with other entities in the models. This is usually called the 'accessibility relation', and there are four types. Their function is to determine which worlds are possible to which. And by that determination alone, we can know why different modal systems give different answers to the same question concerning necessity (Cf. Kripke, 1971: 63-72).

Nevertheless, while the most important pioneer of this kind of model-theoretic semantics, Saul Kripke, characterizes his theory in a way tending to cause us to think of some entities in the manner we think of this world, he does not in fact commit himself to believe in any particular kind of entities; and, to the best of my knowledge, neither does he explicitly claim that there are actually other worlds than ours. So, according to this kind of modal semantics, the meanings of modal discourses can merely be elucidated at the level of formal systems. Taking especially our desire to grasp the meanings in more concrete manner into consideration, though it can somehow unify our understanding of the distinct systems, it seemingly still leaves us in doubt as to how to judge among those 'accessibility relations', and which system endorsing them is the most plausible one. Merely because, at the purely formal level, we would not clearly see what definitely are the things, as oppose to just the 
manipulatively constructed symbols, being in those formal relations, and, hence, an ability to decide on the basis of their qualified meanings. We might consider these set of problems as, according to Alvin Plantinga's terminology, problems about 'pure semantics' of modal logic, 'pure' because they give us only some calculustype of meanings of the modal languages (Cf. Chihara, 1998: 115-16). In the next section, I will discuss ways to solve the problem by introducing more concrete ways of interpreting pure semantics, which can be called 'applied semantics' of modal logic.

\section{Meanings of possible worlds}

Though there is a lack of clarity in the model-theoretic interpretation of modal logic, many philosophers consider it a useful apparatus for bringing light to many virtually unsolvable, by other means, philosophical issues, for instance, problems concerning universal properties, the meaning of 'proposition', the question "what is a counterfactual statement?", issues involving laws of nature, or even the existence of God, etc (Cf. Lewis, 1986: Ch. 1). Since the time it first appeared, possible worlds-talk has gradually become fasionable philosophical jargon, treated as if we have common understanding of it. Nevertheless, as I have said, the need to make their contents concrete and clearer has, all along, been acknowledged as well.

Where formal languages for those problematic modal words is concerned, many more concrete, or applied, semantics of possible worlds have been proposed to make sense of any number of axioms, rules of inference, and units of language that different systems of modal logic use; but the contents of those semantics seem to be so self-contained as to bring about many meanings of 'possible worlds' - the concept to be understood first in our attempt to understand the semantics. The alleged meanings of 'possible worlds' consist of a variety of concepts, both having been in use previously in other philosophical topics and offered originally, to which question after question has been put. It is impossible here to enumerate all of those objections and elucidate all the difficulties which each of them confronts; but some of them will be mentioned in the following line of argument.

However, among various ways of dealing with the very concept of possible worlds, it appears to me that none has been in the position that can be properly called 'the lone winner'. Each of them faces a variety of problems peculiar to their way of engaging with the concepts of necessity and possibility. A common characteristic bringing them into problems is their selfunderstanding that they are able to explain the modalities in the way conforming to our everyday understanding of the concepts in question. The problematic theories of possible worlds roughly mentioned below are those of David Lewis, Alvin Plantinga, and Robert Adams.

\section{Lewis' extreme realism}

The 'modal realism' of David K. Lewis (Cf. Lewis, 1973 and 1986) understands possible worlds as a set of entities their natures being the same as that of this world, except only that things inhibit in, or events occur in, them are more or less different from ours. The actual world, however, is just one amongst possible worlds, infinite in number; and is called 'actual' only on the basis that we are calling it while we ourselves are being in it, just as we call here 'here' only because it is where we are now. In other words, every possible world is in 
fact 'actual', in a sense that they all exist as equally concrete objects; but their coexistences are not connected by space and time, otherwise we would understand them to be the same world or universe. If we say that it is possible that George W. Bush is elected, instead, as the president of China, Lewis would mean it as saying that there is at least one world in which another person so similar to 'our' Bush that can be equally called 'George W. Bush' (or as Lewis himself understands, as 'Bush's counterpart') is elected as the president of China in that other world.

We can easily see why this theory of possible worlds is a focus of many objections; his theory of possible worlds is trying to articulate that there are actual other-worldly worlds, in which I, you, George Bush, or every thing we know exist, but that we can not causally be in contact with. Moreover, he is trying to tell us that despite such separation of its existing from us, they are things we actually refer to when we talk modally. So it is not surprising that few philosophers, if any, actually endorse this position. Lewis himself seems to accept that his position is prone to objections; but he considers those objections as 'the incredulous stare', using only 'common sense' as the basis for rejecting his view. He thinks that if we consider the theoretical advantages his theory can offer, we will see that his way is the best way to analyze modality, because it can reduce, as he claims, all concepts involving modal notions in our modal talks to concrete things or objects that just exist, even if their existence are so exotic.

I think no one would blame us if we dismiss this exotic modal theory of Lewis, but I do not think that we can equally dismiss his strong intention to propose a modal theory that has real explanatory power - power to make us grasp the meanings of modal discourses without making use of other modal concepts. As we will see, other existing possible worlds theories discussed below do not qualify as an adequate theory of necessity because of their inability to meet such explanatory standards that Lewis has set.

\section{Plantinga's modal Platonism}

The second theory concerning the nature of possible worlds is that of Alvin Plantinga (Cf. Plantinga, 1979). He treats possible worlds as abstract entities called 'state of affairs'. It would better help us to sense what this state of affairs is like by thinking of Plato's 'world of ideas'. Thus we could call this theory 'Modal Platonism'.

A state of affairs could either obtain or not obtain. A state of affairs that obtains is the same one as that which we call 'actual'; and this actual world is understood by Plantinga to be 'the maximal' set of non-contradicted states of affairs that obtains. He believes that this idea conforms more satisfactorily to our intuition involving modality than the realism of Lewis; by reason that it does not refer to things which are not of this world, but attempts to talk about entities belonging to only the actual world, so he calls his theory 'modal actualism'. Nevertheless, this actualist theory could be seen to be not actualistic as it claims, for we can equally suspect the existence of such states of affairs as that of Lewis's other concrete universes. It is not easy nowadays to persuade ourselves to believe both that Plato's world of forms really exists and that its existence is essential to our discussion about necessity.

One additional problem of this kind of possible worlds theory is that we have to accept that we can not use modal theory for 
expositing all kinds of our modal discussion. As we saw, according to this theory, we have to understand possible worlds as one obtainable: one with, at least, a possibility to obtain. This involvement of other modal notions in the process of analyzing modal discourses, as mentioned in 3.1 and which will be discussed further below, is problematic to a theory which could be called an adequate modal theory.

\section{Possible worlds as propositions}

The last possible worlds theory to be considered here is that of Robert Adams, treating possible worlds as maximal noncontradictory sets of propositions, and the actual world as a maximal set of true propositions (Cf. Adams, 1979: 253-73). Its main problem is comparable to that of Plantinga's concerning its explanatory power, because the propositions in the set have to be those which could have been true; which is also a modal notion. Furthermore, it tries to base an aspect of propositions, the modal aspect, on other aspects of themselves, which could be considered as reinforcing the problematic situation just mentioned, rather than helping to solve it.

Consequently, I would like to find some new way to understand the problematic concept of necessity in a manner that makes our ability to grasp the concept its important element. One feasible way I would like to suggest here is of understanding modal concepts in causal terms. I am quite positive that it more directly points to what really is meant by the words "necessary" and "possible". When I say "It is possible that Hitler is still alive", I am neither thinking that there does exist someone similar to him in at least another world whose existence is independent in every aspect from ours, nor referring to some state of affairs, in which there is the 'essence' of Hitler, that exists without its obtaining. I also do not think that appealing to some kind of proposition will help us to grasp the real meaning of that talk. What I mean by the above statement is this: I know that in fact Hitler is dead. Moreover, I know how he died. But I believe that the fact of his dying is not a necessary fact, as it might not have happened. A question that can be put to me is "Why do you believe that?" One answer I can give is "Because Germany might not have been defeated". The role my answer plays in relation to my belief in question is an explanatory role, by which the content of my belief could be elucidated. In which manner does the answer explain the initial statement? Obviously, one, and perhaps the commonly believed, of the events forcing Hitler to decide to kill himself was that the Germans were being defeated; that is, the Germans' being defeated was one of the causes of Hitler's death. A modal statement, then, receives an essential part of its content from contents of a statement that is able to be considered as one having a causal relation to the things said in the modal statement. This examination, I think, takes us back to the basic idea that lies behind any occasion of using modal words. And we should try to redo our work starting from this idea.

In the following sections I try to make feasible the project of grounding modal concepts on causality by, first, examining some problems and constraints of understanding both concepts, then, trying to get involved in the semantics of possible worlds through giving a kind of applied semantic in some figurative form. Finally, I will compare my proposed way with some others. 


\section{A realist theory of causality}

A crucial problem that we need to consider first is the problem about the definition of 'cause'. If, in our attempt to understand modal concepts, we have to give a particular definition of the concept, a constraint is to be kept in mind: it should be a definition which can be accepted generally. But it seems that in doing so we might not have to fix our understanding of the concept 'cause' on any particular meaning; in addition, there may be justifications in treating it as a primitive concept. The reason for this will be mentioned later.

As mentioned earlier, there is another constraint, and in my opinion it is the more crucial one, that is: in the process of understanding modal concepts by causal concepts we must try to exclude, as much as possible, the concepts to be understood from the definition of concepts to which we appeal. Our task seems to be a troublesome one as far as this constraint is concerned, since modal concepts have traditionally been used in defining what 'cause' is by many philosophers, from the Greeks down to contemporaries. Fortunately, there are at least some philosophers who question that way of defining 'cause' and give an alternative way to grasp the relation between a cause and its effect. One such philosopher is G. E. M. Anscombe (Anscombe, 1996: 20423). By combining Anscombe-type causality with a belief, that is 'the causal principle', tacitly held by virtually all of us, the present project will have a future.

In her work, Anscombe gives an account of causation which could be described, in her own words, as 'radically different'. It differs from the 'traditional' accounts of causation of, e.g., Aristotle, Spinoza,
Hume, Kant, or Mill, that associates the notion of cause with necessity in one way or another. To put it briefly, a cause has been considered as something necessarily preceding its effect; or in another formulation, the effects of the same kind are always determined by and invariably succeed the causes of the same kind, either logically or physically. As Hume saw, understanding causation in this way has created a difficulty: the supposed necessity and generality could not be found in situations in which we think the relation between cause and its alleged effect has occurred. For this reason, Anscombe wrote:

[...] it's not difficult to show it prima facie wrong to associate the notion of cause with necessity or universality in this way. For, it being much easier to trace effects back to causes with certainty than to predict effects from causes, we often know a cause without knowing whether there is an exeptionless generalization of the kind envisaged, or whether there is a necessity". [Then she added] "...knowledge of causes is possible without any satisfactory grasp of what is involved in causation [...] (Anscombe, 1996: 208)

After that, she stated what she thought the basic meaning of causality was:

"Causality consists in the derivativeness of an effect from its causes... Effects derive from, arise out of, come of, their causes" (Anscombe, 1996: 209). 
Anscombe's last statement was seen by her to be the one 'that lies under our noses' forgotten by analysis in terms of necessity. However, even if it is the case that all of us already share this notion of causality, it might not be sufficient to use this shared notion to understand possibility in terms of causation. Unless we can claim that everything that is able to be expressed in modal statements is also able to have some kind of causal relation according to that common notion, we will not be able to get a truly workable way of grounding possibility. One way to deal with this constraint is to find some causal notion shared by all of us about everything. I see at least one notion that can help us here. It is called 'the causal principle' or 'the principle of causality'. The content of this notion is that every event, every change, and every occurrence of something has a cause. Though it is still in doubt whether we can rationally defend this principle, it seems to me that our belief in it is deep and wide enough to take it for granted. It is not difficult to notice that there is something in Anscombe's account of causality that is much congruent with the idea of the causal principle, namely both see one side of the relatas - say, an effect - as the pivot of the notion. This fact simultaneously reinforces the credibility of Anscombe's account. Now, if it is plausible to believe in the causal principle, the common notion about causality as Anscombe explains and as we have seen, an explanatory power of causal statements by which a modal statement could be brought to light; understanding possibility in causal terms might also be more plausible.

To illustrate my point and prepare ourselves to touch the possible worlds issues in the next section, let me give a sketch of how we can have a model to understand causality from the account Anscombe has given us. Anscombe argued that we have knowledge of causality partly from the fact that many causal concepts are already represented in many words of our language (Anscombe, 1996: 210). This is one of the reasons why, if there is no proper way to understand cause, we eventually might use this fact in justifying treating causation as a primitive concept.

Anscombe gave an example in which the cause of certain positions and movements of a ball is to be identified. One conclusion showed by the example is that we can not always deduce the effect from the cause (Anscombe, 1996: 213). This is partly because, even if an effect is always determined, its being determined may be in various ways, in her words " ...to give content to the idea of something's being determined, we have to have a set of possibility, which something narrows down to one - before the event" (Anscombe, 1996: 216). Then, we can believe that everything is determined without any commitment to determinism. Another reason for not believing in the deducibility of an effect from a cause is that there are many different sorts of causality (Anscombe, 1996:220).

She has given examples of two main types of cause in connection to necessity (I think that what the examples show is an understanding of necessity using the concept of causation rather than the contrary.) that, at the same time, can partly serve our purpose too. The first is called 'necessitating cause' explained as follows: "a cause $\mathrm{C}$ is a necessitating cause of an effect $\mathrm{E}$ when (I mean: on the occasions when) if $\mathrm{C}$ occurs it is certain to cause $\mathrm{E}$ unless something prevents it. [or it is not possible (on the occasions) that $\mathrm{C}$ should 
occur and should not cause an E, given that there is nothing that prevents an $\mathrm{E}$ from occurring.] The second one is "nonnecessitating cause' explained as "one that can fail of its effect without the intervention of anything to frustrate it" (Anscombe, 1996: 219). An example of the first one is that rabies is the cause of death; and of the second is that a certain reading of the Geiger counter registers and some radioactive material are the cause of the explosion of a bomb connected.

From the above explanation, we have seen that the conditional interference and prevention can block our understanding of necessitation considered as necessitation simpliciter; that is, necessity can be understood only under some condition, and it is a causal one. Notice further that in saying that in the context an effect being determined a set of possibilities must be presupposed, possibility concerned here should not be the unconditional possibility, say, it must be relevant to the matter of causation. There is a statement that seems to support this: "That this effect was produced by this cause does not at all show that it could not, or would not, have been produced by something else in the absence of this cause" (Anscombe, 1996: 220) [the emphasis is mine]. I think that somehow we must treat 'cause' as a primitive concept if we want to use it in understanding modal notions. At least, Anscombe, seemingly, has given us a reason for doing so. As she wrote: "...the causation itself is, one could say, mere hap. It is difficult to explain this idea any further" (Anscombe, 1996: 220).

\section{Towards a causal theory of necessity}

Now we have the material to construct a more formal model of understanding modal notions via causality. Barring the possibility that the fashionable possible worlds semantics and tools of set theory are so troublesome that we can no longer use it, I would like to show that it is possible to identify possible worlds with sets of causal conditions of some sort. We assume that everything has a cause, hence a thing that has the status of a 'cause' of something itself also has a cause, and the procedure goes on in this way, perhaps ad infinitum. We, then, can have a set of events or things being a part of the entire procedure. We can call events or things in that set the members of the set. Each member of the set has at least a relation with another event or thing belong to the same set. The relation in question is some kind of causal relation. So, we can understand the ability of each member to be a member of some set that it is the ability to be in a causal relation, and we have assumed that everything has this relation by nature. It is possible that there are an infinity of such kinds of sets. But as we have seen, they are possible by virtue of the causal characteristics they have. If we think that causation has a temporal direction, we might add that those sets must be ordered sets. Therefore, we can give a condition of being a different set: set $\mathrm{A}$ is a different set from set $\mathrm{B}$ if and only if $\mathrm{A}$ has at least a member that is different from $\mathrm{B}$, or its members are ordered differently from B. If this actual world is the aggregation of every event that occurred in it, every change that takes place in it, and everything existing in it; this actual world could be identified with one of such sets. But it is specially different from others in the sense that this set has a concrete exemplification. Then, possible worlds can be identified with 'possible'-as opposed to possible simpliciter-sets that somehow different from the only-oneexemplified set.

Putting it in a more formal manner, we can formulate a figurative form of the formal 
definitions of three main modal concepts as follows:

A statement, $p$, is a possible one if and only if the content stated in $\mathrm{p}$ stands in some causal relations with that of other statements, or, alternatively, a statement, $\mathrm{p}$, is a possible one if and only if there is a causal system $S_{1}$ such that $p$ is a member of $\mathrm{S}_{1}$

A statement, q, is a necessary one if and only if the content stated in q stands in any and all causal relations with that of any other statements, or, alternatively, a statement, $\mathrm{p}$, is a necessary one if and only if $\mathrm{q}$ is a member of all and any causal system $S_{n}$ for all $n$ which is a member of the set of natural numbers

A statement, $r$, is an actual one if and only if the content stated in $r$ stands in some causal relations with that of other statements and these relation do obtain in this world, or, alternatively, a statement, $r$, is an actual one if and only if there is a causal system $S_{a}$ such that $r$ is a member of $\mathrm{S}_{\mathrm{a}}$, where $\mathrm{S}_{\mathrm{a}}$ is a system that obtains in this world.

Concerning the constraint of being able to understand de re, and de dicto modality (modality concerning Thing referred to in the statement and concerning the statement itself, respectively), we must be able to cope with it by our framework. We can identify 'thing' or 'object' in possible worlds by beginning to look at the causal status things or objects in this actual world have. We can identify the 'property' of an object in possible worlds in the same way. If we can cope with de re modality, being able to understand de dicto seems to follow. This is yet to be worked out. ${ }^{4}$

\section{Conclusion}

In this last section I will compare my proposed project with others' on some selected issues as following:

(1) My 'possible worlds' might be considered by David Lewis as, in his own term, 'the ersatz worlds'. Because they have no existence as this actual world has. It is, in fact, merely one of the ways of talking about or of understanding modal concepts. But it is, I think, one promising way too.

(2) My 'possible worlds' might be considered as Actualist's worlds because they are able to be constructed from things and the supposed relations they have in this actual world. But it seems distinct from, and perhaps has some privilege over, another actualistic version, such as Plantinga's, partly because of the fact that it accommodates better our understanding of 'possible objects' -ones that do not have their existence in the actual world but are merely possible. Let me explain. Suppose we believe that it is possible that there is a hundred fingers man, and we believe that

\footnotetext{
4 I have elaborated a more full-form modal theory in this realist vein elsewhere. That theory is much larger in its scope and detail so cannot be presented in these limited pages. Nevertheless, it is different from the theory I presented here in that: I went far beyond treating 'causality' as simply a primitive concept that needs no further elaboration. I employed ideas from the work of Michael Tooley (Cf. for example Tooley, 1987), who could be called a 'realist' concerning causality, to construct my own modal theory (Bunnag, 2003).
} 
having the ten fingers of an ordinary man must have some cause, perhaps determined by something about human chromosomes, then, we can think about some possible causal sets in which at least one of its members has approximately a pattern of causal relations with other things like ours, enough so that it can be called 'human', but some conditions taking part in determining of the number of fingers is different from ours, so that 'he' is caused to have a hundred fingers. Even though that merely possible man does not really exist, via this proposed way of understanding we can grasp that in what way such a man is possible.

(3) In comparing the possible worlds as both a state of affairs and as the other worlds like ours, possible worlds understood in terms of causation seems less difficultly than the former ways. At least, we are more accustomed to causal concepts than to the stipulated concept of state of affairs or Lewisian's worlds that do not have any causal relation to ours. If we want to discuss the truth of modal statements with someone holding a belief in those stipulated concepts, we can go no further than his assertion. But is it not the case that in our discussion we think that there is somehow a way to, or are able to, settle the argument? Grounding modal truth on the truth about causality can keep our belief about the nature of our discussion, because, at least, we can hope that science is able to give us some answers about that kind of truth.

\section{References}

Adams, Robert Merrihew. 1979.

"Theories of Actuality" in The

Possible and the Actual. Michael

J. Loux (ed.). Cornell University

Press. Pp. 253-73.

Anscombe, G. E. M. 1996. "Causality and

Determination" in Women

Philosophers. Mary Warnock (ed.).

Everyman. Pp. 204-03.

Bunnag, Puttawit. 2003. An Investigation of The Metaphysics of Modality. unpublished Ph.D.Dissertation, Department of Philosophy, Faculty of Arts, Chulalongkorn University.

Chihara, Charles S. 1998. The Worlds of Possibility: Modal Realism and the Semantics of Modal Logic. Oxford: Oxford University Press.

Hintikka, Jaakko. 1979 "The Modes of Modality" in The Possible and the Actual. Michael J. Loux (ed.). Cornell University Press. Pp. 65-79.

Huges, G. E. and Cresswell, M. J. 1996. A New Introduction to Modal Logic. Routledge.

Kripke, Saul. 1971. "Semantical Considerations on Modal Logic" in Reference and Modality.Leonard Linsky (ed.). Clarendon Press. Pp. 63-72.

Lewis, David K. 1973. Counterfactuals. Blackwell Publishers.

Lewis, David K. 1986. On the Plurality of Worlds. Blackwell Publishers.

Loux, Michael J. (ed.) 1979. The Possible and the Actual. Cornell University Press.

Plantinga, Alvin. 1979. "Actualism and Possible Worlds" in The Possible and the Actual. Michael J. Loux (ed.). Cornell University Press. Pp. 253-73. 